TRANSACTIONS OF THE

AMERICAN MATHEMATICAL SOCIETY

Volume 359, Number 1, January 2007, Pages 447-463

S 0002-9947(06)03969-9

Article electronically published on August 24, 2006

\title{
EXTENSION AND SEPARATION PROPERTIES OF POSITIVE DEFINITE FUNCTIONS ON LOCALLY COMPACT GROUPS
}

\author{
EBERHARD KANIUTH AND ANTHONY T. LAU
}

\begin{abstract}
Continuing earlier work, we investigate two related aspects of the set $P(G)$ of continuous positive definite functions on a locally compact group $G$. The first one is the problem of when, for a closed subgroup $H$ of $G$, every function in $P(H)$ extends to some function in $P(G)$. The second one is the question whether elements in $G \backslash H$ can be separated from $H$ by functions in $P(G)$ which are identically one on $H$.
\end{abstract}

\section{INTRODUCTION}

Let $G$ be a locally compact group and let $P(G)$ be the set of continuous positive definite functions of $G$. Let $H$ be a closed subgroup of $G$. Following the terminology of [28, we call $H$ an extending subgroup of $G$ if every continuous positive definite function on $H$ extends to some continuous positive definite function on $G$. This is equivalent to requiring that the restriction map between the Fourier-Stieltjes algebras $B(G)$ and $B(H)$ (compare 8 ) is surjective. We say that $G$ has the extension property if each closed subgroup of $G$ is extending.

For any closed subgroup $H$ of $G$, let

$$
P_{H}(G)=\{\phi \in P(G): \phi(h)=1 \text { for all } h \in H\} .
$$

Slightly modifying the notation of [20] and 21], we call $H$ a separating subgroup if for every $x \in G \backslash H$, there exists $\phi \in P_{H}(G)$ such that $\phi(x) \neq 1$. Also, $G$ is said to have the separation property if each closed subgroup of $G$ is separating. The interest in and importance of the separation properties arose from the fact that it turned out to be useful in studying the ideal theory of the Fourier algebra $A(G)$ (see 20]).

The extension and the separation properties have been studied by several authors (see [5], 7], [9], 13, 14, [19], 24] and [28] for the extension property and [20, 21] and 25] for the separation property). If $H$ is a closed subgroup of $G$ such that $G$ has small $H$-conjugation invariant neighbourhoods of the identity $\left(G \in[S I N]_{H}\right)$, then $H$ is extending as well as separating ([7, [10] and [13]). Consequently, every group with small conjugation invariant neighbourhoods (SIN-group) satisfies both the extension and the separation property. Conversely, if $G$ is either almost connected

Received by the editors June 30, 2004 and, in revised form, February 2, 2005.

2000 Mathematics Subject Classification. Primary 43A35; Secondary 22E25.

Key words and phrases. Locally compact group, positive definite function, extension, separation property, solvable group, almost connected group, SIN-group.

The second author was supported by NSERC grant A 7679 .

(C)2006 American Mathematical Society 
or a compactly generated nilpotent group, then either of these properties forces $G$ to be a SIN-group (see [7, [15, [19] and [20]). Also, for $G$ almost connected, the separating subgroups of $G$ have been identified as precisely the so-called neutral subgroups [21] (for the notion and basic theory of neutral subgroups see [23] and 32]). Very recently, Losert [25] has given an intricate characterization of neutral subgroups of locally compact groups that possess an almost connected open normal subgroup. In addition, Losert pointed out the connection between the separation property and the Mautner phenomenon (see [31] and 34]).

However, apart from these many similarities between the extension and the separation properties, there are marked differences between them as soon as single subgroups or locally compact groups which are neither nilpotent nor almost connected are considered. For instance, normal subgroups are of course always separating, whereas Douady's observation (see [8, p. 204] and [16, (34.28)]) shows that an abelian normal subgroup of a connected group is extending only if it is contained in the centre. In this context, it is worthwhile to mention that the main result of [7] provides necessary and sufficient conditions for a positive definite function on a closed normal subgroup of a locally compact group $G$ to be extendible to $G$. Moreover, there are 2-step solvable groups which satisfy one of these properties but not the other (see Section 4 for details).

In this paper we further investigate the extension and separation properties. In Sections 2 and 3 we focus on the question of when all infinite closed cyclic subgroups are extending or separating. For brevity of notation, we say that $G$ has the extension property for cyclic subgroups (respectively, the separation property for cyclic subgroups) if every closed subgroup of $G$ which is isomorphic to $\mathbb{Z}$ is extending (respectively, separating). Note that the extension and the separation properties for cyclic subgroups are local properties in the sense that a locally compact group has one of these properties if and only if every compactly generated open subgroup has the corresponding property. It turns out that for almost connected groups and for compactly generated nilpotent groups $G$, the separation property for cyclic subgroups implies that $G$ is a SIN-group (Theorem 2.7 and Theorem 3.5). In contrast, every nilpotent locally compact group has the extension property for cyclic subgroups (Theorem 3.2).

It was observed in 20, Remark 2.6] that if $G$ is nilpotent and $G$ has the extension property, then it also has the separation property. This does not remain true for solvable groups. In fact, in Section 4 we shall show that the Fell group, which is an extension of the additive group of $p$-adic numbers by a compact multiplicative group of $p$-adic numbers, satisfies the separation property but not the extension property. In Section 5 we determine all the extending subgroups of examples such as the $a x+b$-group and the motion group of the plane. Finally, in Section 6 we add a number of propositions which demonstrate that some of our results cannot be generalized.

\section{Preliminaries on the eXtension Property}

Let $G$ be a locally compact group and let $P^{1}(G)=\{\phi \in P(G): \phi(e)=1\}$. Then $P^{1}(G)$ is a convex set, and $\operatorname{ex}\left(P^{1}(G)\right)$ will denote the set of extreme points of $P^{1}(G)$. For $\phi \in P(G)$, we denote by $\pi_{\phi}$ the associated cyclic unitary representation of $G$ and, for any representation $\pi$ of $G$, by $\mathcal{H}(\pi)$ the Hilbert space of $\pi$. 
Let $H$ be a closed subgroup of $G$ and let $\varphi \in P(H)$. If $\varphi$ extends to some element in $B(G)$, then it also extends to some function in $P(G)$ [28, Theorem 3.3]. If $\tau$ and $\pi$ are representations of $H$ and $G$, respectively, then $\operatorname{ind}_{H}^{G} \tau$ will denote the representation of $G$ induced by $\tau$ and $\left.\pi\right|_{H}$ the restriction of $\pi$ to $H$.

The following lemma is something one would expect as a basic result in the area. However, since we could not find a reference, we include a proof for the reader's convenience.

Lemma 1.1. Let $H$ be a closed subgroup of a locally compact group $G$ and let $\varphi \in$ $P^{1}(H)$. Then $\varphi$ extends to some $\phi \in P(G)$ if and only if $\pi_{\varphi}$ is a subrepresentation of $\left.\pi\right|_{H}$ for some representation $\pi$ of $G$. If $\pi_{\varphi}$ is irreducible, then $\pi$ can be chosen to be irreducible.

Proof. If $\pi_{\varphi}$ is a subrepresentation of $\left.\pi\right|_{H}$, then there exists an isometric linear mapping $U$ from $\mathcal{H}\left(\pi_{\varphi}\right)$ into $\mathcal{H}(\pi)$ such that $U \pi_{\varphi}(h)=\pi(h) U$ for all $h \in H$. It follows that, for some $\eta \in \mathcal{H}\left(\pi_{\varphi}\right)$ and all $h \in H$,

$$
\varphi(h)=\left\langle\pi_{\varphi}(h) \eta, \eta\right\rangle=\left\langle U \pi_{\varphi}(h) \eta, U \eta\right\rangle=\langle\pi(h) U \eta, U \eta\rangle .
$$

Thus $\phi$, defined by $\phi(x)=\langle\pi(x) U \eta, U \eta\rangle(x \in G)$, is a continuous positive definite function extending $\varphi$.

Conversely, let $\phi \in P(G)$ be an extension of $\varphi$. Then $\phi(x)=\langle\pi(x) \xi, \xi\rangle, x \in G$, for some representation $\pi$ of $G$, namely $\pi=\pi_{\phi}$, and some $\xi \in \mathcal{H}(\pi)$. Now, $\varphi(h)=$ $\left\langle\pi_{\varphi}(h) \eta, \eta\right\rangle, h \in H$, where $\eta$ is a cyclic vector for $\pi_{\varphi}$. Since $\mathcal{H}\left(\pi_{\varphi}\right)$ is the closed linear span of all $\pi_{\varphi}(h) \eta, h \in H$, the mapping

$$
\sum_{j=1}^{n} c_{j} \pi_{\varphi}\left(h_{j}\right) \eta \rightarrow \sum_{j=1}^{n} c_{j} \pi\left(h_{j}\right) \xi\left(h_{j} \in H, c_{j} \in \mathbb{C}, n \in \mathbb{N}\right)
$$

extends to an isometric linear mapping $U$ of $\mathcal{H}\left(\pi_{\varphi}\right)$ into $\mathcal{H}\left(\pi_{\phi}\right)$. Finally, $U$ satisfies

$$
U \pi_{\varphi}(h) \pi_{\varphi}(k) \eta=\pi(h k) \xi=\pi(h)(\pi(k) \xi)=\pi(h)\left(U \pi_{\varphi}(k) \eta\right)
$$

for all $h, k \in H$, whence $U \pi_{\varphi}(h)=\pi(h) U$, as required.

For the last statement we only have to note that if $\pi_{\varphi}$ is irreducible, then $\varphi \in$ $\operatorname{ex}\left(P^{1}(H)\right)$ and by [14, Proposition 2, p. 275] there exists $\phi \in \operatorname{ex}\left(P^{1}(G)\right)$ extending $\varphi$.

Remark 1.2. Let $N$ be a closed normal subgroup of a locally compact group $G$. Then $G$ acts on $N$ by inner automorphisms and hence on positive definite functions and on representations in the obvious manner. Let $\varphi \in \operatorname{ex}\left(P^{1}(N)\right)$ and suppose that $\varphi$ extends to some element of $P(G)$. Then the stabilizer $G_{\pi_{\varphi}}=\left\{y \in G: y \cdot \pi_{\varphi}=\pi_{\varphi}\right\}$ of $\pi_{\varphi}$ is open in $G$. Indeed, $\|y \cdot \varphi-\varphi\|_{B(N)} \rightarrow 0$ as $y \rightarrow e$ and, on the other hand, $\|y \cdot \varphi-\varphi\|_{B(N)}=2$ whenever the irreducible representations $\pi_{\varphi}$ and $\pi_{y \cdot \varphi}=y \cdot \pi_{\varphi}$ are inequivalent (see [5, Lemma 2]). Consequently, the subgroup $G_{\pi_{\varphi}}$ of $G$ contains a neighbourhood of $e$.

Lemma 1.3. Let $G$ be a locally compact group and $N$ a closed normal subgroup of finite index. If $H$ is a closed subgroup of $G$ such that $H \cap N$ is extending in $N$, then $H$ is extending in $G$.

Proof. Let $\psi \in P(H), K=H \cap N$ and $\varphi=\left.\psi\right|_{K}$. By hypothesis, there exists $\phi \in P(N)$ such that $\left.\phi\right|_{K}=\varphi$. We claim that the representation $\rho=\operatorname{ind}_{N}^{G} \pi_{\phi}$ has the property that $\left.\rho\right|_{H} \geq \pi_{\psi}$. 
To see this, note first that since $H / K$ is finite,

$$
\pi_{\psi} \leq \operatorname{ind}_{K}^{H} \pi_{\varphi}=\operatorname{ind}_{K}^{H} \pi_{\left.\phi\right|_{K}} \leq \operatorname{ind}_{K}^{H}\left(\left.\pi_{\phi}\right|_{K}\right) .
$$

On the other hand, for any representation $\tau$ of $N$,

$$
\operatorname{ind}_{K}^{H}\left(\left.\tau\right|_{K}\right) \leq\left.\left(\operatorname{ind}_{N}^{G} \tau\right)\right|_{H}
$$

Indeed, since $K=H \cap N$ and $G / N$ is finite, it is easily verified that the range of the map

$$
T: \mathcal{H}\left(\operatorname{ind}_{K}^{H}\left(\left.\tau\right|_{K}\right)\right) \rightarrow\{F: G \rightarrow \mathcal{H}(\tau)\}
$$

defined by $T \xi(x)=0$ if $x \notin H N$ and $T \xi(x)=\tau\left(y^{-1}\right) \xi(h)$ if $x=h y$ with $h \in H$ and $y \in N$, is contained in

$$
\mathcal{H}\left(\operatorname{ind}_{N}^{G} \tau\right)=\mathcal{H}\left(\left.\left(\operatorname{ind}_{N}^{G} \tau\right)\right|_{H}\right)
$$

and that $T$ provides an intertwining operator between $\operatorname{ind}_{K}^{H}\left(\left.\tau\right|_{K}\right)$ and $\left.\left(\operatorname{ind}_{N}^{G} \tau\right)\right|_{H}$. Thus $\pi_{\psi} \leq\left.\left(\operatorname{ind}_{N}^{G} \pi_{\phi}\right)\right|_{H}$, and therefore by Lemma $1.1 \psi$ extends to some element of $P(G)$.

\section{Almost connected groups}

Let $G$ be a locally compact group and let $H$ and $K$ be closed subgroups of $G$ such that $h^{-1} K h \subseteq K$ for all $h \in H$. Then $K$ is said to have small $H$-invariant neighbourhoods ( $K$ belongs to the class $[S I N]_{H}$ ) if $K$ has a neighbourhood basis $\mathcal{V}$ of the identity such that $h^{-1} V h=V$ for all $V \in \mathcal{V}$ and $h \in H$. If $G \in[S I N]_{G}$, then $G$ is called a SIN-group. SIN-groups and their structure have been extensively studied by Grosser and Moskowitz [12].

Recall that a locally compact group $G$ is said to have the separation property for cyclic subgroups if every closed subgroup of $G$ which is isomorphic to $\mathbb{Z}$ is separating in $G$. Our first purpose is to show that an almost connected group must be a SIN-group whenever it has the separation property for cyclic subgroups. This strengthens Theorem 1.1 of 20 . Some parts of the proof are analogous to those of [20, Theorem 1.1], so that we only have to indicate the necessary alterations. The main new ingredient, however, is Theorem 1 of [25]. The separation property for cyclic subgroups is less handy than the full separation property since we do not know whether it passes to arbitrary quotient groups. Yet we have

Lemma 2.1. Let $H$ be a locally compact group and $C$ a compact normal subgroup of $H$ such that $H / C$ is either almost connected or locally connected. If $H$ has the separation property for cyclic subgroups, then $H / C$ has the corresponding property.

Proof. Let $B$ be a closed subgroup of $H / C$ which is isomorphic to $\mathbb{Z}$ and let $q$ : $H \rightarrow H / C$ denote the quotient homomorphism. Choose $a \in H$ such that $q(a)$ generates $B$ and let $A$ denote the closed subgroup of $H$ generated by $a$. Then $A$ cannot be compact and hence is isomorphic to $\mathbb{Z}[16$, Theorem 9.1], and $q(A)=B$. Let $\mathcal{U}$ be a neighbourhood basis of the identity in $H$. Since $H$ has the separation property for cyclic subgroups, $A=\bigcap\{\overline{A U A}: U \in \mathcal{U}\}$ [21, Theorem 1.3]. Using the compactness of $C$, it is straightforward to verify that $q(\overline{A U A})=\overline{B q(U) B}$ and

$$
\bigcap\{q(\overline{A U A}): U \in \mathcal{U}\}=q(\bigcap\{\overline{A U A}: U \in \mathcal{U}\}) .
$$


Thus it follows that

$$
\begin{aligned}
B & =q(A)=q(\bigcap\{\overline{A U A}: U \in \mathcal{U}\})=\bigcap\{q(\overline{A U A}): U \in \mathcal{U}\} \\
& =\bigcap\{\overline{B q(H U) B}: U \in \mathcal{U}\} .
\end{aligned}
$$

Since the set of all $q(U), U \in \mathcal{U}$, forms a neighbourhood basis of the identity in $H / C, B$ satisfies condition (ii) of [21, Theorem 1.3]. Since $H / C$ is either almost connected or locally connected, $B$ is a separating subgroup of $H / C$ 21, Theorem $1.3]$.

Lemma 2.2. Suppose that $G$ contains a central torus $\mathbb{T}^{d}$ such that $G / \mathbb{T}^{d}$ is a vector group. If $G$ has the separation property for cyclic subgroups, then $G$ is the direct product of $\mathbb{T}^{d}$ and a vector group.

Proof. As in the proof of Lemma 1.5 of [20], assuming that $G$ fails to be abelian, it suffices to consider the reduced Heisenberg group. In this case, retaining the notation of [20, replace the group $L$ by the subgroup $\{(n, 0,0): n \in \mathbb{Z}\}$, which turns out to be not separating.

Lemma 2.3. Let $G$ be a semidirect product $G=A \ltimes V$ where $V$ is a vector group and $A$ is a connected locally compact group. If $G$ has the separation property for cyclic subgroups, then $G$ is the direct product of $A$ and $V$.

Proof. Let $\alpha: A \rightarrow \operatorname{Aut}(V), a \rightarrow \alpha_{a}$ denote the automorphism defined by

$$
\left(e, \alpha_{a}(y)\right)=(a, 0)(e, y)(a, 0)^{-1}, y \in V, a \in A .
$$

We have to show that $\alpha_{a}(y)=y$ for all $y \in V$ and $a \in A$. Suppose that $\alpha(A)(y) \neq$ $\{y\}$ for some $y \in V$ and let $D=\mathbb{Z} y$. Since $D$ is separating, by [25, Theorem 1] there exists a compact neighbourhood $U$ of the identity in $G$ such that $d U d^{-1}=U$ for all $d \in D$. Thus, for all $a \in A$ and $x \in V$ satisfying $(a, 0) \in U$ and $(e, x) \in D$,

$$
\left(a, x-\alpha_{a}(x)\right)=(e, x)(a, 0)(e, x)^{-1} \in U .
$$

Now, there exists $a \in A$ such that $(a, 0) \in U$ and $\alpha_{a}(y) \neq y$. Indeed, otherwise we have $\alpha_{a}(y)=y$ for all $a \in A$ since $A$ is connected. This is a contradiction. Since every automorphism of a vector group is linear, we then obtain $\left(a, n\left(y-\alpha_{a}(y)\right)\right) \in U$ for all $n \in \mathbb{Z}$. This contradicts the compactness of $U$.

Corollary 2.4. Let $G$ be a solvable simply connected Lie group, and suppose that $G$ has the separation property for cyclic subgroups. Then $G$ is abelian.

Proof. Proceeding by induction, we can assume that the statement of the lemma holds for simply connected solvable Lie groups $H$ with $\operatorname{dim} H<\operatorname{dim} G$. Choose a connected closed normal subgroup $N$ of $G$ of codimension one. Then $G$ is isomorphic to a semidirect product $\mathbb{R} \ltimes N$. By the inductive hypothesis, $N$ is a vector group and Lemma 2.3 implies that $G$ is abelian.

Corollary 2.5. Let $G$ be a locally compact group containing a closed normal vector subgroup $V$ such that $G / V$ is compact and connected. If $G$ has the separation property for cyclic subgroups, then $G$ is the direct product of $V$ and a compact group.

Proof. Since $V$ is a vector group and $G / V$ is compact, there exists a compact subgroup $K$ of $G$ such that $G$ is a semidirect product of $K$ and $V$ [17, Theorem VIII]. The lemma is now an immediate consequence of Lemma 2.3. 
Combining Lemma 2.2 and Corollaries 2.4 and 2.5 and using Lemma 2.1, the next lemma follows in exactly the same way as on page 95 of $[20$.

Lemma 2.6. Let $G$ be a solvable connected Lie group and suppose that $G$ has the separation property for cyclic subgroups. Then $G$ is the direct product of a compact abelian group and a vector group.

Now we are in a position to prove the theorem announced at the beginning of this section.

Theorem 2.7. Let $G$ be an almost connected locally compact group. If $G$ has the separation property for cyclic subgroups, then $G$ is a SIN-group.

Proof. Note first that $G$ is a projective limit of Lie groups [29, Theorem 4.6] and a projective limit of SIN-groups is a SIN-group. Since the separation property for cyclic subgroups passes to quotients modulo compact normal subgroups (Lemma 2.1 ), we can assume that $G$ is a Lie group. Then, since $G / G_{0}$ is finite, it suffices to show that $G_{0} \in[S I N]$. Hence we can assume that $G$ is a connected Lie group.

Let $R$ denote the radical of $G$ and $q: G \rightarrow G / R$ the quotient homomorphism. The following reasoning to show that $G / R$ is compact is analogous to that in the proof of [7, Corollary 2]. First of all, since $R$ has the separation property for cyclic subgroups, it is the direct product of a vector group and a compact abelian group (Lemma 2.6). If $G / R$ is non-compact, it has an Iwasawa decomposition $G / R=K A N$, where $K$ is compact, $A$ is abelian and $N$ is nilpotent. The group $A$ normalizes but does not centralize $N$. Then $q^{-1}(A N)$ is a connected solvable Lie group having the separation property for cyclic subgroups. By Lemma 2.6, $q^{-1}(A N)$ is abelian. In particular, $A N$ is abelian, which is a contradiction. Thus $G / R$ is compact.

As in the last but one paragraph of the proof of Theorem 1.1 in [20, pages 95/96] it follows that $G$ possesses a closed normal vector subgroup $V$ such that $G / V$ is compact and connected. Applying Corollary 2.5 again, we conclude that $G$ is the direct product of a vector group and a compact group.

\section{NiLPotent GROUPS}

In this section we study the extension property for cyclic subgroups and the separation property for cyclic subgroups for nilpotent groups. It turns out that nilpotent groups always have the extension property for cyclic subgroups whereas the separation property for cyclic subgroups, like the full separation property, proves to be very restrictive.

An element $x$ of a locally compact group is called compact if the subgroup generated by $x$ is relatively compact. Let $G^{c}$ denote the set of all compact elements of $G$. In general, of course, $G^{c}$ fails to be a subgroup of $G$. However, if $G$ is nilpotent, then $G^{c}$ is a closed subgroup of $G$ [11.

Lemma 3.1. Let $G$ be a locally compact group such that $G_{0}$ is nilpotent. Let $A$ be a closed cyclic subgroup of $G$ and suppose that there exists an almost connected open subgroup $L$ of $G$ such that $A \cap L \neq\{e\}$. Then $A$ is extending in $G$.

Proof. Let $a$ be a generating element of $A$ and choose $m \in \mathbb{N}$ such that $a^{m} \in L$. Define an open subgroup $M$ of $G$ by $M=\bigcap\left\{a^{j} L a^{-j}: 0 \leq j \leq m-1\right\}$. Then it is easy to check that $A$ normalizes $M$ and hence $H=A M$ is an open subgroup of $G$, and $H / G_{0}$ is compact. It suffices to show that $A$ is extending in $H$. 
Since $H$ is almost connected, it is a projective limit of Lie groups. Fix a compact normal subgroup $K$ of $H$ such that $H / K$ is a Lie group. Then $(H / K)_{0}$ has finite index in $H / K$ and $G_{0} K / K \subseteq(H / K)_{0}$. However, since

$$
(H / K) /\left(G_{0} K / K\right)=H / G_{0} K
$$

is totally disconnected, $(H / K)_{0}=G_{0} K / K$, which is nilpotent. Moreover, since $A \cap K=\{e\}, A$ is topologically isomorphic to $A K / K$. Thus, passing to $H / K$, we can assume that $H$ is a Lie group and $H_{0}$ is nilpotent and has finite index in $H$.

Let $B=A \cap H_{0}$. Then $B \cap H_{0}^{c}=\{e\}$, and since $H_{0} / H_{0}^{c}$ is a connected and simply connected nilpotent Lie group, it follows from [19, Lemma 3.3] that $B$ is extending in $H_{0}$. Lemma 1.3 now shows that $A$ is extending in $H$.

In [19, Lemma 3.3] it was observed that if $G$ is a connected and simply connected nilpotent Lie group, then $G$ has the extension property for cyclic subgroups. The next theorem and its corollary extend this considerably. We shall see later (Proposition 6.4) that such a result fails for simply connected solvable Lie groups with trivial centre.

Theorem 3.2. Let $G$ be a locally compact group such that $G_{0}$ is nilpotent. In addition, suppose that every compactly generated open subgroup $H$ of $G$ possesses an almost connected open subgroup which is normal in $H$. Then $G$ has the extension property for cyclic subgroups.

Proof. Let $A$ be a closed cyclic subgroup of $G$ and choose a compactly generated open subgroup $H$ of $G$ containing $A$. By hypothesis, there exists an almost connected open subgroup $L$ of $H$ which is normal in $H$. If $A \cap L \neq\{e\}$, then $A$ is extending in $G$ by Lemma 3.1. Thus, assume that $A \cap L=\{e\}$. Then, since $L$ is open and normal in $H, A=A L / L$, and therefore $A$ is extending in $H$ and hence also in $G$.

In passing we remind the reader that a locally compact group $G$ is called locally nilpotent if, given any compact subset $C$ of $G$, the closed subgroup of $G$ generated by $C$ is nilpotent. Obviously, the class of locally nilpotent groups is much larger than the class of nilpotent groups.

Corollary 3.3. Let $G$ be a locally compact group such that $G_{0}$ is nilpotent and $G / G_{0}$ is locally nilpotent. Then $G$ has the extension property for cyclic subgroups.

Proof. We only have to observe that $G$ satisfies the hypothesis of the preceding theorem. For that, let $H$ be any compactly generated open subgroup of $G$ and let $L=G_{0} H^{c}=H_{0} H^{c}$. Then $L$ is almost connected since $H^{c}$ is compact [11] and $H_{0} H^{c}$ is open in $H$ [1].

We are now turning to the separation property for cyclic subgroups. In the proof of Theorem 3.5 below, we shall need the following simple lemma, which of course is a very special case of Theorem 2 of $[25$. We believe it is appropriate to avoid the use of the deep Theorem 2 of [25] whenever possible.

Lemma 3.4. Let $G$ be a semidirect product $G=D \ltimes G_{0}$, where $D$ is discrete and $G_{0}$ is locally connected. If $D$ is separating in $G$, then $G_{0} \in[S I N]_{D}$.

Proof. By Theorem 1.3 of [21, $D$ is neutral. Thus given any neighbourhood $U$ of the identity in $G_{0}$, there exists a neighbourhood $V$ of the identity such that $V \subseteq U$ 
and $D V=V D$. We claim that $a V a^{-1}=V$ for all $a \in D$. Fix $a$; then for every $x \in V$ there exist $y_{x} \in V$ and $b_{x} \in D$ such that $a x=y_{x} b_{x}$ or $a x a^{-1}=y_{x}\left(b_{x} a^{-1}\right)$. Now, since $G_{0}$ is connected, $a G_{0} a^{-1} \subseteq G_{0}$ is connected, whence $b_{x}=a$ for all $x \in V$. This shows that $a x a^{-1} \in V$ for all $x \in V$.

In the proofs of the next theorem and of Proposition 6.2 we shall use the following fact about $[S I N]_{H}$-groups.

Let $A, B$ and $M$ be closed subgroups of a locally compact group $G$ such that $B$ and $M$ are normal and $M \in[S I N]_{A} \cap[S I N]_{B}$. Then $M \in[S I N]_{A B}$. This can be seen as follows. Let $U$ be a neighbourhood of the identity in $M$ such that $a U a^{-1}=U$ for all $a \in A$. There exists a neighbourhood $V$ of the identity satisfying $V \subseteq U$ and $b V b^{-1}=V$ for all $b \in B$. Let $W=\bigcup_{a \in A} a V a^{-1}$. Then $W \subseteq U$ and, since $B$ is normal in $G, A B$ is a subgroup, and it follows from the construction of $W$ that $c W c^{-1} \subseteq W$ for all $c \in A B$.

Theorem 3.5. Suppose that $G$ contains a compactly generated nilpotent closed normal subgroup $N$ such that $G / N$ is compact. If $G$ has the separation property for cyclic subgroups, then $G$ is a SIN-group.

Proof. Since $N$ is nilpotent and compactly generated and $G / N$ is compact, $G$ is a projective limit of Lie groups [18, Theorem 9]. By Lemma 2.1, $G / C$ has the separation property for cyclic subgroups for any compact normal subgroup $C$ such that $G / C$ is a Lie group. Moreover, a projective limit of SIN-groups is a SIN-group. Therefore, to prove the theorem we can assume that $G$ is a Lie group. Note that a locally compact group is a SIN-group whenever it has a subgroup of finite index which is a SIN-group.

Since $G_{0}$ has the separation property for cyclic subgroups, $G_{0}$ is a SIN-group by Theorem 2.7. Let $q: G \rightarrow G / G_{0}$ be the quotient homomorphism. Since $G / G_{0}$ is discrete and $G / N$ is compact, $q(N)$ is a nilpotent subgroup of finite index in $G / G_{0}$. Thus we can assume that $q(N)=G / G_{0}$. In addition, since $N$ is compactly generated, $G / G_{0}$ is finitely generated and hence has a finitely generated torsion free subgroup of finite index [3, Theorem 2.1]. Again, after passing to a subgroup of finite index, we can therefore assume that $G / G_{0}$ is a finitely generated torsion free nilpotent group. It follows that $G$ has a sequence of normal subgroups $G_{0} \subseteq$ $G_{1} \subseteq \ldots \subseteq G_{r}=G$ with the property that $G_{j} / G_{j-1}$ is isomorphic to $\mathbb{Z}, 1 \leq j \leq r$. So $G_{j}=A_{j} \ltimes G_{j-1}, 1 \leq j \leq r$, where $A_{j}$ is isomorphic to $\mathbb{Z}$. Since $G$ has the separation property for cyclic subgroups, $G_{0} \in[S I N]_{A_{j}}$ by Lemma $3.4,1 \leq j \leq r$. This implies that $G$ is a SIN-group. Indeed, since $G_{0}$ is a SIN-group, this follows by induction on $j$ using what we have observed before the theorem.

In the sequel, for subsets $A$ and $B$ of a group $G$, let $[A, B]$ denote the set of commutators $a b a^{-1} b^{-1}, a \in A, b \in B$.

Lemma 3.6. Let $H$ be an almost connected nilpotent locally compact group. If $H$ has the separation property for cyclic subgroups, then $H_{0}$ is contained in the centre of $H$.

Proof. Since $H$ is almost connected it is a projective limit of Lie groups $H / C_{\alpha}$ [29, Theorem 4.6]. For each $\alpha$, let $q_{\alpha}: H \rightarrow H / C_{\alpha}$ denote the quotient homomorphism. Then $q_{\alpha}\left(H_{0}\right) \subseteq\left(H / C_{\alpha}\right)_{0}$ for every $\alpha$ and hence $H_{0} \subseteq \bigcap_{\alpha}\left(\left(H / C_{\alpha}\right)_{0}\right)$. Suppose we have shown that $\left(H / C_{\alpha}\right)_{0}$ is contained in the centre of $H / C_{\alpha}$ for every $\alpha$. Then, for $x \in H_{0}$ and $y \in H,[x, y] \in C_{\alpha}$ for all $\alpha$, and hence $[x, y]=e$, as required. 
Since by Lemma 2.1 the separation property for cyclic subgroups passes to the quotients $H / C_{\alpha}$, we can therefore assume that $H$ is a Lie group. So $H / H_{0}$ is finite and $H_{0}$ is a connected Lie group. Since $H_{0}$ has the separation property for cyclic subgroups, $H_{0}=V \times K$, where $V$ is a vector group and $K$ is a compact connected Lie group (Theorem 2.7). Moreover, since $K$ is nilpotent, it follows that $K=\mathbb{T}^{d}$ for some $d \in \mathbb{N}_{0}$.

Let $a \in H$ and let $A$ denote the closed subgroup of $H$ generated by $a$. Since $H$ has the separation property for cyclic subgroups, $H_{0}$ normalizes $A$ [21, Lemma 2.3]. Using the facts that $H_{0}$ and $A$ are abelian and that $H_{0}$ normalizes $A$, it is easily verified by induction that

$$
\left[y^{m}, a\right]=[y, a]^{m}=\left[y, a^{m}\right]
$$

for all $y \in H_{0}$ and $m \in \mathbb{N}$. Fix $q \in \mathbb{N}$ such that $a^{q} \in H_{0}$, and let $x$ be an arbitrary element of $H_{0}$. Since $V$ and $\mathbb{T}^{d}$ are divisible, there exists $y \in H_{0}$ such that $y^{q}=x$. Then

$$
[x, a]=\left[y^{q}, a\right]=\left[y, a^{q}\right]=e .
$$

This shows that $H_{0}$ is contained in the centre of $H$.

Theorem 3.7. Let $G$ be a locally nilpotent locally compact group and suppose that $G$ has the separation property for cyclic subgroups. Then $G_{0}$ is contained in the centre of $G$.

Proof. As before, let $G^{c}$ denote the subgroup of all compact elements in $G$. Then $G_{0} G^{c}$ is an open normal subgroup of $G$ and $G / G_{0} G^{c}$ is torsion free [11].

Let $a$ be any element of $G \backslash G_{0} G^{c}$ and let $A$ denote the closed subgroup of $G$ generated by $a$. Then $A \cap G_{0} G^{c}=\{e\}$ and $A$ is discrete, hence isomorphic to $\mathbb{Z}$. Let $H$ be any compactly generated open subgroup of $G$ such that $a \in H$. Then $H$ is nilpotent and has the separation property for cyclic subgroups. By 21, Lemma 2.3], $H_{0}=G_{0}$ normalizes $A$. So $\left[G_{0}, a\right] \subseteq A$, and since $A$ is discrete and $\left[G_{0}, a\right]$ is connected, we obtain that $\left[G_{0}, a\right]=\{e\}$.

Now, let $a \in G_{0} G^{c}$ and let $H$ denote the closed subgroup generated by $a$ and $G_{0}$. Then $H$ is nilpotent and $H / G_{0}$ is compact. Since $H$ has the separation property for cyclic subgroups, by Lemma $3.6 G_{0}$ is contained in the centre of $H$. Combining the two cases, we conclude that $G_{0}$ is contained in the centre of $G$.

Corollary 3.8. Let $G$ be a compactly generated nilpotent locally compact group. Then the following conditions are equivalent:

(i) $G$ has the separation property.

(ii) $G$ has the separation property for cyclic subgroups.

(iii) $G$ is a projective limit of groups each of which has an open centre.

(iv) $G$ is a SIN-group.

Proof. Notice first that (iii) implies (iv) because a projective limit of SIN-groups is again a SIN-group. Therefore, it only remains to prove (ii) $\Rightarrow$ (iii).

Since $G$ is compactly generated, it is a projective limit of Lie groups $G / C_{\alpha}[18$. By Lemma 2.1, the separation property for cyclic subgroups passes to the quotients $G / C_{\alpha}$. Since $G / C_{\alpha}$ is a Lie group, $\left(G / C_{\alpha}\right)_{0}$ is open in $G / C_{\alpha}$, and by Lemma 3.6, $\left(G / C_{\alpha}\right)_{0}$ is contained in the centre of $G / C_{\alpha}$. Thus (iii) holds. 
It is not unlikely that in Corollary 3.8 the hypothesis that $G$ be compactly generated can be dropped. Yet, at present we have no idea of how to treat totally disconnected nilpotent groups.

\section{The Fell GROUP}

Losert [25] has announced an example of a locally compact group $G$ such that $G$ has a compact open normal subgroup and every proper closed subgroup of $G$ is compact, but $G$ is not a SIN-group. This example shows that even within the class of IN-groups (that is, groups which have at least one compact conjugation invariant neighbourhood of the identity) there exist groups which may have both the separation property and the extension property without being a SIN-group. Recall that in 14 an example was given of a compactly generated 2-step solvable Lie group which is not an IN-group and nevertheless has the extension property, but not the separation property. This group is the semidirect product $G=\mathbb{Z} \ltimes \mathbb{R}$, where $n \in \mathbb{Z}$ acts on $\mathbb{R}$ by multiplication with $2^{n}$. It is easily seen that the subgroup $\mathbb{Z}$ is not separating. We now present an example of a 2 -step solvable group which has the separation property, but not the extension property, and fails to be an IN-group.

Let $p$ be a prime number and let $N$ be the additive group of the $p$-adic number field $\mathbb{Q}_{p}$. Let $K$ be the multiplicative group of $p$-adic numbers of valuation one. Form the semidirect product $G=K \ltimes N$ where $K$ acts on $N$ by multiplication. This group $G$ is usually referred to as the Fell group. It is the most prominent example of a non-compact locally compact group with countable dual (see 11). We are going to show the following.

Theorem 4.1. Let $G$ be the Fell group. Then

(i) $G$ has the separation property.

(ii) A closed subgroup of $G$ is extending (if and) only if it is compact or open.

Let $H$ be a closed subgroup of $G$. We have verified in 21, Example 3.2(ii)] that $H$ is separating if and only if $H$ is either compact or $H \supseteq N$. We claim that there are no other closed subgroups. In particular, this proves that $G$ has the separation property.

To start with, let $H$ be a closed subgroup of $G$ such that $N \nsubseteq H$. We have to show that $H$ is compact. Then $H \cap N$ is either trivial or a proper closed subgroup of $N$ and as such is compact. Indeed, every non-trivial proper closed subgroup of $N$ is of the form $p^{k} \mathbb{Z}_{p}$, where $\mathbb{Z}_{p}$ is the subring of $p$-adic integers and $k \in \mathbb{Z}$ (see [16, (10.16)(a)]). Moreover, $H / H \cap N$ is abelian since the homomorphism $H / H \cap N \rightarrow G / N=K$ is injective. Let $q: G \rightarrow G / N$ denote the quotient homomorphism. We claim that $q(H)$ is closed in $K$. To see this, let $\left(a_{n}, x_{n}\right) \in H$, $n \in \mathbb{N}$, such that $a_{n} \rightarrow a$ for some $a \in K$. Assume that $q(H) \neq\{1\}$ and fix $(b, y) \in H$ such that $b \neq 1$. Since $H / H \cap N$ is abelian, there exists a sequence $\left(z_{n}\right)_{n}$ in $H \cap N$ such that

$$
\begin{aligned}
\left(a_{n} b, x_{n}+a_{n} y\right) & =\left(a_{n}, x_{n}\right)(b, y)=\left(1, z_{n}\right)(b, y)\left(a_{n}, x_{n}\right) \\
& =\left(b a_{n}, z_{n}+y+b x_{n}\right) .
\end{aligned}
$$

Since $K$ is abelian, we get that

$$
x_{n}+a_{n} y=z_{n}+y+b x_{n} .
$$


Moreover, since $H \cap N$ is compact, we can assume that $z_{n} \rightarrow z$ for some $z \in H \cap N$. It follows that

$$
(1-b) x_{n}=z_{n}+\left(1-a_{n}\right) y \rightarrow z+(1-a) y .
$$

Thus $x_{n}$ converges to $x=(1-b)^{-1}(z+(1-a) y) \in N$. Hence $\left(a_{n}, x_{n}\right) \rightarrow(a, x)$ in $H$, whence $a \in q(H)$.

Finally, since $H$ is $\sigma$-compact and $q(H)$ is closed in $K$, the group isomorphism between $H / H \cap N$ and $H N / N=q(H)$ is a homeomorphism. Thus $H$ is compact since both $H \cap N$ and $H / H \cap N$ are compact.

Turning to (ii), by what we have just shown, it remains to consider the case that $H$ contains $N$. Note first that the abelian normal subgroup $N$ is not extending in $G$. To see this, fix a character $\omega$ of $N$ with kernel equal to $\mathbb{Z}_{p}$. Then the map $x \rightarrow \omega_{x}$, where $\omega_{x}(y)=\omega(x y)$ for $y \in N$, is a topological isomorphism between $N$ and $\widehat{N}$. Since $(k \cdot \omega)(y)=\omega(k y)$ for all $k \in K$ and $y \in N$, it follows that the stabilizer of any non-trivial character of $N$ is trivial. However, by Douady's observation (see [16, (34.48)(d)] or [8]), a character of an abelian normal subgroup $A$ of a locally compact group $B$ extends to a continuous positive definite function on $B$ only if its stabilizer is open in $B$. Thus $N$ is not extending.

Now, suppose that $H$ properly contains $N$, that is, $H=C \ltimes N$ for some nontrivial closed subgroup $C$ of $K$. We claim that $C$ is either open in $K$ or finite. Let

$$
U=\left\{x \in N:|x|_{p}<p^{-1 /(p-1)}\right\}
$$

and

$$
V=\left\{y \in K:|x-1|_{p}<p^{-1 /(p-1)}\right\} .
$$

Then $U$ is an open subgroup of $N$ and $V$ is an open subgroup of $K$ of index $p-1$. The exponential map

$$
x \rightarrow \exp (x)=\sum_{j=0}^{\infty} \frac{x^{j}}{j !}
$$

forms a topological isomorphism from $U$ onto $V$ (see [22, p. 80, Proposition]). Thus, if $C \cap V$ is non-trivial, then $C \cap V$ is open in $K$ since $\log (C \cap V)$ is a nontrivial subgroup of $N$ and hence is open in $N$. If $C \cap V$ is trivial, then $C$ is finite. In the first case $H$ is open in $G$, while in the second case $N$ is open in $H$ and therefore $H$ cannot be extending.

Summarizing what we have seen, we obtain that a closed subgroup of $G$ is extending if and only it is compact or open in $G$.

\section{EXAMPLES}

We begin with two lemmas which are useful in determining the extending subgroups.

Lemma 5.1. Suppose that $G$ has a closed normal subgroup $N$ such that, with $q: G \rightarrow G / N$ denoting the quotient homomorphism,

$$
B(G) \subseteq B(G / N) \circ q+C_{0}(G) .
$$

Let $H$ be a non-compact closed subgroup of $G$. If $H$ is extending, then $H \cap N \subseteq$ $[H, H]$. 
Proof. Towards a contradiction, suppose there exists $h \in H \cap N$ with $h \notin[H, H]$. Select a character $\chi$ of $H$ such that $\chi(h) \neq 1$. By hypothesis, $\chi$ extends to some $\phi \in P(G)$ and $\phi=\phi_{1}+\phi_{2}$, where $\phi_{1}$ is constant on cosets of $N$ and $\phi_{2}$ vanishes at infinity. Then, since $h \in N$,

$$
\phi(x h)=\phi_{1}(x)+\phi_{2}(x h)
$$

for all $x \in G$, and, since $|\phi(h)|=1$,

$$
\phi(x h)=\phi(x) \phi(h)=\chi(h)\left(\phi_{1}(x)+\phi_{2}(x)\right) .
$$

It follows that

$$
(1-\chi(h)) \phi_{1}(x)=\chi(h) \phi_{2}(x)-\phi_{2}(x h)
$$

for all $x \in G$. Now, the function $x \rightarrow \chi(h) \phi_{2}(x)-\phi_{2}(x h)$ vanishes at infinity, and hence so does $\phi_{1}$ since $\chi(h) \neq 1$. Therefore $\phi=\phi_{1}+\phi_{2}$ vanishes at infinity, whence $\chi=\phi \mid H$ vanishes at infinity on $H$. Since $\chi$ is a character, this forces $H$ to be compact, a contradiction.

Lemma 5.2. Let $G$ be a locally compact group and let $N$ be a non-compact closed normal subgroup of $G$ such that $G / N$ is compact, abelian and second countable. Suppose that

$$
B(G) \subseteq B(G / N) \circ q+C_{0}(G)
$$

If $H$ is an abelian closed subgroup of $G$ and $H$ is extending, then $H$ must be compact.

Proof. Let $\chi$ be a character of $H$ and let $\phi \in P(G)$ be an extension of $\chi$. Write $\phi=\phi_{1}+\phi_{2}$, where $\phi_{1} \in B(G / N) \circ q$ and $\phi_{2} \in C_{0}(G)$. Then, as in the proof of Lemma 5.1,

$$
\phi_{1}(x h)-\chi(h) \phi_{1}(x)=\chi(h) \phi_{2}(x)-\phi_{2}(x h)
$$

for all $h \in H$ and $x \in G$. Since the function $x \rightarrow \phi_{1}(x h)-\chi(h) \phi_{1}(x)$ is constant on $N$, whereas $x \rightarrow \chi(h) \phi_{2}(x)-\phi_{2}(x h)$ vanishes at infinity, and since $N$ is noncompact, it follows that

$$
\phi_{1}(x h)=\chi(h) \phi_{1}(x) \text { and } \phi_{2}(x h)=\chi(h) \phi_{2}(x)
$$

for all $x \in G$ and $h \in H$.

Now assume that $H$ is non-compact. Since the function $h \rightarrow \phi_{2}(x h)$ vanishes at infinity on $H$ and its absolute value equals $\left|\phi_{2}(x)\right|$, we obtain that $\phi_{2}(x)=0$ for all $x \in G$. Hence $\phi=\phi_{1}$ and so $\phi \in P(G / N) \circ q$. By [14, Proposition 2, p. 275], we can assume that $\phi \in \operatorname{ex}\left(P^{1}(G)\right)$ and hence $\phi \in \operatorname{ex}\left(P^{1}(G / N)\right) \circ q=\widehat{G / N} \circ q$ (since $G / N$ is abelian). Thus we have shown that $\left.\widehat{H} \subseteq(\widehat{G / N} \circ q)\right|_{H}$. But $\widehat{G / N}$ is countable, since $G / N$ is compact and second countable. Therefore $\widehat{H}$ is countable. This contradicts the non-compactness of $H$.

Example 5.3. Let $G$ be the $a x+b$-group, that is, the semidirect product $\mathbb{R}_{+}^{\times} \ltimes \mathbb{R}$, where the multiplicative group of positive real numbers acts on $\mathbb{R}$ by multiplication. We determine all the extending subgroups of $G$. We identify $\mathbb{R}$ with the normal subgroup $\{1\} \times \mathbb{R}$ and $\mathbb{R}_{+}^{\times}$with the subgroup $\mathbb{R}_{+}^{\times} \times\{0\}$. Lemma 5.1 applies to $G$ with $N=\mathbb{R}[27$. It is known that the normal subgroup $\mathbb{R}$ is not extending. Let $H \neq\{e\}$ be an extending subgroup of $G$ and assume first that $H$ is connected. Since $[H, H] \subseteq \mathbb{R}$, by Lemma $5.1, H \cap \mathbb{R}=[H, H]$. Thus, since $[H, H]$ is connected, either $H \cap \mathbb{R}=\mathbb{R}$ or $H \cap \mathbb{R}=\{0\}$. In the first case, $H=\mathbb{R}$ or $H=G$.

Now, let $H$ be an arbitrary extending subgroup of $G$. Since $H_{0}$ is open in $H, H_{0}$ is extending as well. By what we already know, either $H_{0}=\{0\}, H_{0}=G$ or $G$ is the 
semidirect product of $\mathbb{R}$ with $H_{0}$. In the last case, $H_{0}=H$. Indeed, given any $a \in$ $\mathbb{R}_{+}^{\times}$, there is a unique $x_{a} \in \mathbb{R}$ such that $\left(a, x_{a}\right) \in H_{0}$. Now, $(1, y)\left(a, x_{a}\right)(1, y)^{-1}=$ $\left(a, x_{a}+y-a y\right)$, so that $y=a y$ for all $a \in \mathbb{R}_{+}^{\times}$and hence $y=0$ whenever $(1, y)$ normalizes $H_{0}$. It remains to consider the case that $H$ is discrete. Then $H \cap N$ is extending and hence $H \cap N \subseteq[H \cap N, H \cap N]=\{0\}$ by Lemma 5.1. Thus, if $H$ is an extending proper subgroup, then $H \cap \mathbb{R}=\{0\}$.

Conversely, a subgroup $H$ with $H \cap \mathbb{R}=\{0\}$ is extending. To see this, note that since $G / N$ is abelian it can be verified in exactly the same way as for the Fell group in the proof of Theorem 4.1 that $H N$ is closed in $G$. Then, given $\varphi \in P(H)$, define $\psi \in P(H N / N)$ by $\psi(h N)=\varphi(h), h \in H$. Then $\psi$ extends to some $\phi \in P(G / N)$ and $\phi \circ q$ extends $\varphi$. Thus $H$ is extending.

Summarizing, we have seen that the proper extending subgroups of $G$ are precisely the closed subgroups $H$ of $G$ such that $H \cap \mathbb{R}=\{0\}$. In [21, Example 3.2(i)] we have seen that the only separating subgroups of $G$ are the normal subgroups. In particular, $G$ and $\{e\}$ are the only subgroups of $G$ which are both extending and separating.

The hypothesis of Lemma 5.1 is also satisfied by simple Lie groups with finite centre [33] and by motion groups, that is, semidirect products $S O(d) \ltimes \mathbb{R}^{d}(d \geq 2)$ (see [6] and 2]). For both kinds of groups we have determined all the separating subgroups in 21]. However, we are unable to specify the extending subgroups, except for the motion group of the plane. Therefore we confine ourselves to this special case.

Example 5.4. Let $G=S O(2) \ltimes \mathbb{R}^{2}$, where $S O(2)$ acts on $\mathbb{R}^{2}$ by rotation, and identify $\mathbb{R}^{2}$ with the normal subgroup $N=\left\{(E, x): x \in \mathbb{R}^{2}\right\}$, where $E$ is the unit matrix. We claim that a proper closed subgroup of $G$ is extending only if it is compact. For that, note first that no non-trivial subgroup of $\mathbb{R}^{2}$ can be extending since $B(G) \subseteq B(G / N) \circ q+C_{0}(G)$.

Now let $H$ be a proper extending subgroup of $G$. Since $H_{0}$ is open in $H, H_{0}$ is extending as well. By Lemma 5.1, $H_{0} \cap \mathbb{R}^{2}=\left[H_{0}, H_{0}\right]$, which is connected. So $H_{0} \cap \mathbb{R}^{2}$ is either equal to $\{0\}$ or $\mathbb{R}^{2}$ or $\mathbb{R} v$ for some $v \in \mathbb{R}^{2}, v \neq 0$. If $H \supseteq \mathbb{R}^{2}$, then, since $H$ is proper, $H_{0}=\mathbb{R}^{2}$, which fails to be extending. Next, let $H_{0} \cap \mathbb{R}^{2}=\mathbb{R} v$. Then $H_{0}=\mathbb{R} v$, since $E$ and $-E$ are the only elements of $S O(2)$ mapping $\mathbb{R} v$ to itself. However, $\mathbb{R} v$ is not extending. Finally, let $H_{0} \cap \mathbb{R}^{2}=\{0\}$. Then either $H_{0} \mathbb{R}^{2}=G$ or $H_{0}$ is trivial. In the first case, $H_{0}$ is compact and from $H_{0} \cap \mathbb{R}^{2}=\{0\}$ and $H_{0} \mathbb{R}^{2}=G$ it is easily deduced that $H_{0}$ is its own normalizer. In particular, $H_{0}=H$. In the second case $H$ is discrete and hence $H \cap \mathbb{R}^{2}$ is extending. Thus $H \cap \mathbb{R}^{2}=\{0\}$, and hence $H$ is abelian. Now Lemma 5.2 shows that $H$ is finite. This finishes the proof of the above claim. Thus a proper closed subgroup of $G$ is extending if and only if it either finite or conjugate to $S O(2)$.

\section{Some AdDitional RESUlts}

In this final section we present some propositions that show limitations to possible extensions of our results. Recall that the Fell group, which has the separation property, is a compact extension of an abelian normal subgroup $N$. Proposition 6.1 in particular shows that such a group cannot have the extension property unless it is a SIN-group. Also, observe that $N$ is not compactly generated. A consequence 
of Proposition 6.2 is that if $G$ is a compact extension of a compactly generated abelian group and $G$ is not a SIN-group, then the separation property fails for $G$.

In the following two propositions we shall consider compact extensions of Moore groups. Recall that a locally compact group $H$ is called a Moore group if all the irreducible representations of $H$ are finite dimensional. These groups are named after C.C. Moore, who completely clarified their structure [30]. In particular, Moore groups are SIN-groups.

Proposition 6.1. Let $G$ contain a closed normal subgroup $N$ such that $N$ is a second countable Moore group and $G / N$ is compact. If $N$ is extending in $G$, then $G$ is a Moore group.

Proof. It is a standard fact that if $(K, X)$ is a transformation group with $K$ compact and $X$ a $T_{1}$-space, then $K$-orbits in $X$ are closed. Taking $X=\widehat{N}$ and $K=G / N$ and observing that $\widehat{N}$ is second countable, we conclude that Mackey's theory applies to $G$ and the normal subgroup $N$ (see [26] and the references therein). Thus, given an irreducible representation $\pi$ of $G$, there exists $\tau \in \widehat{N}$ such that $\left.\pi\right|_{N}$ is weakly equivalent to the orbit $G(\tau)$ in $\widehat{N}$. Let $\tau=\pi_{\varphi}$, where $\varphi \in \operatorname{ex}\left(P^{1}(N)\right)$. Since $N$ is extending, by Remark 1.2 the stabilizer $G_{\tau}=\{x \in G: x \cdot \tau=\tau\}$ of $\tau$ is open in $G$ and hence of finite index. By Mackey's theory, $\pi=\operatorname{ind}_{G_{\tau}}^{G} \sigma$ for some $\sigma \in \widehat{G_{\tau}}$ such that $\left.\sigma\right|_{N}$ is a multiple of $\tau$. Then $\operatorname{dim} \tau=\left[G: G_{\tau}\right] \operatorname{dim} \sigma$, and it therefore suffices to show that $\sigma$ is finite dimensional.

By Mackey's theory of multiplier representations [26], $\sigma$ can be obtained as follows. There exist a multiplier $\omega$ on $G_{\tau} / N$, an (irreducible) $\omega$-representation $\gamma$ of $G_{\tau}$ in $\mathcal{H}(\tau)$ (which extends $\tau$ ) and an irreducible $\bar{\omega}$-representation $\delta$ of $G_{\tau} / N$ such that $\sigma=\gamma \otimes(\delta \circ q), q: G_{\tau} \rightarrow G_{\tau} / N$. Then $\operatorname{dim} \gamma=\operatorname{dim} \tau$ and $\operatorname{dim} \delta<\infty$. Indeed, $\delta$ can be viewed as an ordinary irreducible representation of the central extension defined by $\bar{\omega}$ of the compact group $G_{\tau} / N$. Thus $\sigma$ is finite dimensional.

Proposition 6.2. Let $G$ contain a closed normal subgroup $N$ such that $G / N$ is compact and $N$ is a compactly generated Moore group. If $G$ has the separation property for cyclic subgroups, then $G$ itself is a Moore group.

Proof. We first show that $G$ is a SIN-group. Since $G / N$ is compact and $N$ is compactly generated and every Moore group is a projective limit of Lie groups [30], by [18, Theorem 4] $G$ is a projective limit of Lie groups $G / C_{\alpha}$. Since a projective limit of SIN-groups is a SIN-group, it then suffices to show that each $G / C_{\alpha}$ is a SIN-group. Now, $N C_{\alpha} / C_{\alpha}$ is a normal Moore subgroup of $G / C_{\alpha}$ with a compact quotient. Thus we can assume that $G$ is a Lie group.

Since $G / N$ is compact and $G / G_{0}$ is discrete, $N G_{0} / G_{0}$ has finite index in $G / G_{0}$ and, being a discrete Moore group, itself has an abelian subgroup of finite index 30. Moreover, recall that $G / G_{0}$ is finitely generated. Putting these facts together yields that $G$ possesses a subgroup $H$ containing $G_{0}$ such that $G / H$ is finite and $H / G_{0}=\mathbb{Z}^{d}$ for some $d \in \mathbb{N}_{0}$. Now we find $d$ closed subgroups $A_{1}, \ldots, A_{d}$ of $G$, each isomorphic to $\mathbb{Z}$, such that $A_{j} \cap G_{0}=\{e\}$ and $H=A_{1} \cdot \ldots \cdot A_{d} G_{0}$. Applying Lemma 3.4 to each of the groups $A_{j}$ we conclude that $G_{0} \in[S I N]_{A_{j}}, 1 \leq j \leq d$. Finally, by the paragraph preceding Theorem 3.5 and the fact that $G_{0}$ is a SINgroup (Theorem 2.7), we conclude that $G_{0} \in[S I N]_{H}$. Since $G / H$ is finite, this shows that $G$ is a SIN-group. 
We now apply well-known results in representation theory to conclude that $G$ is actually a Moore group. In fact, since $G$ has a normal subgroup $N$ which is a CCR group and $G / N$ is compact, $G$ itself is a CCR group. Thus $G$ is a type I SIN-group and as such has to be a Moore group.

We have seen (Corollary 3.3) that every nilpotent locally compact group has the extension property for cyclic subgroups. This appears to be special to nilpotent groups as will be demonstrated by Proposition 6.4 below. In preparation for this, we include the next example.

Example 6.3. With the notation of [4, pp. 180-182], let $G=G_{3,4}(\alpha), \alpha \in \mathbb{R}$. Then $G$ can be realized as $\mathbb{R}^{3}$ with multiplication given by

$$
\begin{gathered}
\left(t_{1}, x_{1}, y_{1}\right)\left(t_{2}, x_{2}, y_{2}\right) \\
=\left(t_{1}+t_{2}, x_{1}+e^{\alpha t_{1}}\left(x_{2} \cos t_{1}-y_{2} \sin t_{1}\right), y_{1}+e^{\alpha t_{1}}\left(x_{2} \sin t_{1}+y_{2} \cos t_{1}\right)\right),
\end{gathered}
$$

$t_{j}, x_{j}, y_{j} \in \mathbb{R}, j=1,2$. Let $A=\{(0, n, 0): n \in \mathbb{Z}\}$ and let $\phi \in P(G)$ be such that $\left.\phi\right|_{A}$ is a character of $A$. We are going to show that $\phi(A)=\{1\}$, whence $A$ is not extending in $G$. For $t \in \mathbb{R}$ and $n \in \mathbb{Z}$,

$$
\begin{aligned}
\phi((t, 0,0)) & =\phi([(0, n, 0),(t, 0,0)](t, 0,0)) \\
& =\phi\left(\left(0, n\left(1-e^{\alpha t} \cos t\right),-n e^{\alpha t} \sin t\right)(t, 0,0)\right) .
\end{aligned}
$$

Let $y \in \mathbb{R}$ be arbitrary and let $t_{n}=-y / n, n \in \mathbb{N}$. Then it is easily verified that $-n e^{\alpha t_{n}} \sin t_{n} \rightarrow y$ and $n\left(1-e^{\alpha t_{n}} \cos t_{n}\right) \rightarrow \alpha y$. It follows that

$$
1=\lim _{n \rightarrow \infty} \phi\left(\left(t_{n}, 0,0\right)\right)=\phi((0, \alpha y, y)) .
$$

Thus, for all $t, x \in \mathbb{R}$ and $n \in \mathbb{Z}$,

$$
\begin{aligned}
\phi((t, x, 0)) & =\phi\left((0, n, 0)(t, x, 0)(0, n, 0)^{-1}\right) \\
& =\phi\left(\left(t, x+n\left(1-e^{\alpha t} \cos t\right),-n e^{\alpha t} \sin t\right)\right) \\
& =\phi\left(\left(t, x+n\left(1-e^{\alpha t} \cos t+\alpha e^{\alpha t} \sin t\right), 0\right)\right) .
\end{aligned}
$$

Now, the function $\varphi$ defined by $\varphi(t)=1-e^{\alpha t} \cos t+\alpha e^{\alpha t} \sin t$ is strictly decreasing in the interval $(-\pi / 2,0]$ and strictly increasing in $[0, \pi / 2)$. Let $x<0$, then for sufficiently large $n \in \mathbb{N}$ there exists $t_{n} \in(-\pi / 2, \pi / 2)$ such that $\varphi\left(t_{n}\right)=-x / n$. Necessarily, $t_{n} \rightarrow 0$ as $n \rightarrow \infty$, and hence the above equation yields that

$$
\phi((0, x, 0))=\lim _{n \rightarrow \infty} \phi\left(\left(t_{n}, x+n \varphi\left(t_{n}\right), 0\right)\right)=\phi((0,0,0))=1 .
$$

Of course, then $\phi((0, x, 0))=1$ for all $x \in \mathbb{R}$.

Proposition 6.4. Let $G$ be a simply connected solvable Lie group. If $G$ has the extension property for cyclic subgroups, then the centre of $G$ is non-trivial. Consequently, if every quotient group of $G$ has the extension property for cyclic subgroups, then $G$ is nilpotent.

Proof. Towards a contradiction, suppose the proposition fails to hold and let $G$ be a simply connected solvable Lie group of minimal dimension which has the extension property for cyclic subgroups and nevertheless has a trivial centre. Fix any connected closed normal subgroup $N$ of $G$ of codimension one. Then $Z(N)$ is normal in $G$ and, due to the minimality of $G, Z(N)$ is non-trivial. Actually, $Z(N)_{0}$ is non-trivial because otherwise the discrete normal subgroup $Z(N)$ of the connected group $G$ would be contained in the centre of $G$, contradicting the assumption. Let 
$V \subseteq Z(N)$ be a non-trivial vector subgroup of minimal dimension which is normal in $G$. It is well known that $V$ is of dimension one or two since $G$ is solvable.

Choose any element $a \in G \backslash N$, and let $H$ denote the connected closed subgroup of $G$ generated by $a$ and $V$. Then $\operatorname{dim} V \leq 3$, and the centre of $H$ is trivial since otherwise the centre of $G$ would be non-trivial. Now, such a solvable Lie group either contains the $a x+b$-group as a closed subgroup or is topologically isomorphic to $G_{3,4}(\alpha)$ for some $\alpha \in \mathbb{R} 4$. Since neither of these groups does have the cyclic extension property (Examples 5.3 and 6.3), we have reached a contradiction.

\section{ACKNOWLEDGEMENT}

The authors are indebted to the referee for his valuable comments which led to a number of clarifications.

\section{REFERENCES}

[1] L. Baggett, A separable group having a discrete dual space is compact, J. Funct. Anal. 10 (1972), 131-148. MR0346090 (49:10816)

[2] L. Baggett and K.F. Taylor, Riemann-Lebesgue subsets of $\mathbb{R}^{n}$ and representations which vanish at infinity, J. Funct. Anal. 28 (1978), 168-181. MR0476911 (57:16462)

[3] G. Baumslag, Lecture notes on nilpotent groups, Conf. Ser. in Math. No. 2, Amer. Math. Soc., Providence, RI, 1971. MR0283082 (44:315)

[4] P. Bernat et al., Représentations des groupes de Lie résolubles, Dunod, Paris, 1972. MR0444836 (56:3183)

[5] L.N. Carling, On the restriction map of the Fourier-Stieltjes algebra $B(G)$ and $B_{p}(G)$, J. Funct. Anal. 25 (1977), 236-243. MR.0458051 (56:16254)

[6] C. Chou, Minimally weakly almost periodic groups, J. Funct. Anal. 36 (1980), 1-17. MR0568972 (81f:43009)

[7] M. Cowling and P. Rodway, Restrictions of certain function spaces to closed subgroups of locally compact groups, Pacific J. Math. 80 (1979), 91-104. MR0534697 (80i:43008)

[8] P. Eymard, L'algèbre de Fourier d'une groupe localement compact, Bull. Soc. Math. France 92 (1964), 181-236. MR0228628 (37:4208)

[9] V. Flory, Eine Lebesgue-Zerlegung und funktorielle Eigenschaften der Fourier-StieltjesAlgebra, Inaugural-Dissertation, Heidelberg, 1972.

[10] B. Forrest, Amenability and ideals in $A(G)$, J. Austral. Math. Soc. Ser. A 53 (1992), 143-155. MR.1175708 (93i:43002)

[11] V.M. Gluskov, Locally nilpotent locally bicompact groups, Trudy Moscov. Obshch. 4 (1955), 291-332. (Russian) MR0072422 (17:281b)

[12] S. Grosser and M. Moskowitz, Compactness conditions in topological groups, J. Reine Angew. Math. 246 (1971), 1-40. MR0284541 (44:1766)

[13] R.W. Henrichs, Über Fortsetzung positiv definiter Funktionen, Math. Ann. 232 (1978), 131150. MR0481931 (58:2022)

[14] R.W. Henrichs, On characters of subgroups, Indag. Math. 41 (1979), 273-281. MR0546368 (80i:43009)

[15] R.W. Henrichs, On one-sided harmonic analysis, Proc. Amer. Math. Soc. 80 (1980), 627-630. MR0587940 (82e:43003)

[16] E. Hewitt and K.A. Ross, Abstract harmonic analysis. I, II, Springer-Verlag, Berlin, Heidelberg, New York, 1963, 1970. MR0156915 (28:158) MR0262773(41:7378)

[17] K.H. Hofmann and P.S. Mostert, Splitting in topological groups, Mem. Amer. Math. Soc. 43, American Mathematical Society, Providence, RI, 1963. MR0151544 (27:1529)

[18] K.H. Hofmann, J.R. Liukkonen and W.M. Mislove, Compact extensions of compactly generated nilpotent groups are pro-Lie, Proc. Amer. Math. Soc. 84 (1982), 443-448. MR0640250 (84e:22007)

[19] E. Kaniuth, Extension of positive definite functions from subgroups of nilpotent locally compact groups, Proc. Amer. Math. Soc. 132 (2004), 865-874. MR2019967 (2004k:43013) 
[20] E. Kaniuth and A.T. Lau, A separation property of positive definite functions on locally compact groups and applications to Fourier algebras, J. Funct. Anal. 175 (2000), 89-110. MR:1774852 (2001m:43012)

[21] E. Kaniuth and A.T. Lau, On a separation property of positive definite functions on locally compact groups, Math. Z. 243 (2003), 161-177. MR.1953055 (2003k:43003)

[22] N. Koblitz, p-adic numbers, $p$-adic analysis, and Zeta-functions, Graduate Texts in Mathematics, Vol. 58, Springer-Verlag, Berlin, Heidelberg, New York, 1977. MR0466081 (57:5964)

[23] M. Leischner and W. Roelcke, On neutral subgroups of topological groups, Math. Ann. 282 (1988), 113-129. MR0960836 (90c:22005)

[24] J.R. Liukkonen and W.M. Mislove, Symmetry in Fourier-Stieltjes algebras, Math. Ann. 217 (1975), 97-112. MR0420148 (54:8163)

[25] V. Losert, Separation property, Mautner phenomenon and neutral subgroups. In: Banach algebras and their applications, 223-234, Contemp. Math., 363, Amer. Math. Soc., Providence, RI, 2004. MR2097963

[26] G.W. Mackey, Unitary representations of group extensions, Acta Math. 99 (1958), 265-311. MR0098328 (20:4789)

[27] G. Mauceri, Square integrable representations and the Fourier algebra of a unimodular group, Pacific J. Math. 73 (1977), 143-155. MR0486289(58:6054)

[28] J.R. McMullen, Extensions of positive definite functions, Mem. Amer. Math. Soc. 117, American Mathematical Society, Providence, RI, 1972. MR0445229 (56:3573)

[29] D. Montgomery and L. Zippin, Topological transformation groups, Interscience, New York, 1955. MR0073104 (17:383b)

[30] C.C. Moore, Groups with finite dimensional irreducible representations, Trans. Amer. Math. Soc. 166 (1972), 401-410. MR0302817 (46:1960)

[31] C.C. Moore, The Mautner phenomenon for general unitary representations, Pacific J. Math. 86 (1980), 155-169. MR0586875 (81k:22010)

[32] W. Roelcke and S. Dierolf, Uniform structures on topological groups and their quotients, New York, 1981. MR0644485 (83j:22001)

[33] W.A. Veech, Weakly almost periodic functions on semisimple Lie groups, Monatsh. Math. 88 (1979), 55-68. MR0550072 (81b:22012)

[34] S.P. Wang, On the Mautner phenomenon and groups with property T, Amer. J. Math. 104 (1982), 1191-1210. MR0681733 (84g:22033)

Institut für Mathematik, Universität Paderborn, D-33095 Paderborn, Germany

E-mail address: kaniuth@math.uni-paderborn.de

Department of Mathematical and Statistical Sciences, University of Alberta, EdMONTON, CANADA T6G 2G1

E-mail address: tlau@math.ualberta.ca 